INVESTIGACIÓN

\title{
HONGOS ASOCIADOS AL MATERIAL PARTICULADO, EN OCAÑA NORTE DE SANTANDER
}

\section{FUNGI ASSOCIATED PARTICULATE MATTER, IN OCANA NORTE DE SANTANDER}

Ing. José Arnoldo Granadillo Cuello ${ }^{\mathrm{a}}$, Ing. Alexander Armesto Arenas ${ }^{\mathrm{b}}$, Msc. Juan Carlos Hernández Criado $^{c}$, Jorge Andrés Duarte ${ }^{\mathrm{d}}$, Mayerly Pedraza Felizola ${ }^{\mathrm{e}}$

a,b,c Docentes Investigadores del Programa de Ingeniería Ambiental de la Facultad de Ciencias Agrarias y del Ambiente, Universidad Francisco de Paula Santander Ocaña, Norte de Santander, Colombia. aarmestoa@ufpso.edu.co; jagranadilloc@ufpso.edu.co

d,e Estudiante Investigador del Programa de Ingeniería Ambiental de la Facultad de Ciencias Agrarias y del Ambiente, Universidad Francisco de Paula Santander Ocaña, Norte de Santander, Colombia.jaduarted@ufpso.edu.co; mayerly-0510@hotmail.com

Fecha de recepción: 01-10-2016

Fecha de aprobación: 07-12-2016

Resumen: El objetivo de este trabajo fue caracterizar los hongos presentes en el material particulado como indicador de riesgo de enfermedades respiratorias en Ocaña norte de Santander. Para esto se muestrearon tres filtros extraídos de cada una de las estaciones de la red de monitoreo UFPSO-CORPONOR, se tomaron 5 puntos al azar dentro de cada filtro con dimensiones de $1 \mathrm{~cm}^{2}$ y se inocularon en medio de cultivo agar papa dextrosa (PDA) a $20^{\circ} \mathrm{C}$ durante 15 días, Los resultados mostraron la presencia de 3 géneros de hongos microscópicos: Penicillium sp, Aspergillus níger, Rhizopus sp y hongos unicelulares como las levaduras, que tienen una gran incidencia en enfermedades respiratorias tales como: neumonías, micosis sistémicas, hipersensibilidad y micotoxicosis.

Palabras clave: PM10, enfermedades respiratorias, hongos microscópicos, indicadores de riesgo, agar papa dextrosa.

Abstract: The aim of this study was to characterize the fungi present in the particulate matter as an indicator of risk of respiratory disease in northern Santander Ocaña. For this three filters extracted from each of the stations of the monitoring network Francisco de Paula Santander Ocaña University -CORPONOR were sampled, 5 points were taken randomly within each filter size of $1 \mathrm{~cm} 2$ and inoculated with culture medium potato dextrose agar (PDA) at $20^{\circ} \mathrm{C}$ for 15 days, results showed the presence of three kinds of microscopic fungi: Penicillium $s p$, aspergillus niger, Rhizopus sp and unicellular fungi such as yeast, which have a high incidence 
of respiratory diseases such as pneumonia, systemic fungal infections, hypersensitivity and mycotoxicosis.

Keywords: PM 10, respiratory diseases, microscopic fungi, risk indicators, potato dextrose agar.

\section{INTRODUCCIÓN}

El equilibrio atmosférico se ha alterado por diversas causas naturales y antrópicas, las cuales han cambiado la composición natural del aire. Existe una variedad de factores precursores de este desequilibrio, siendo uno de los más importantes el material particulado producto de las actividades antropogénicas como la producción industrial, los medios de transporte $y$ partículas de polvo suspendidas (García, Agudelo \& Jiménez, 2006). El material particulado es un conjunto de partículas sólidas o líquidas presentes en la atmósfera (Meszaros, 1999), y que pueden estar asociadas a material orgánico como esporas de hongos, bacterias y virus. La importancia del estudio de los niveles, la composición química y microbiológica, así como de las fuentes del material particulado radica en la relación existente con los efectos negativos que en la salud pueda ocasionar este tipo de contaminación. Diversos estudios epidemiológicos demuestran la relación del material particulado presente en el aire y sus microorganismos asociados con la incidencia de enfermedades respiratorias (Oyala \& Pérez, 2006; Jiménez y Cruz, 2006; Minguillon, 2007; Crisóstomo, 2010).

El material particulado se emite principalmente en zonas de actividad urbana, industrial y minera, (Arteaga \&
Duran, 2001). Está dividido en dos grupos principales de acuerdo a su tamaño: PM10 y PM2.5 que corresponden a las partículas con tamaño menor a 10 y $2.5 \mu \mathrm{m}$ (Samara \& Voutsa, 2005). Las partículas menores a 10 $\mu \mathrm{m}$ son sólidas o líquidas, dispersas en la atmósfera como producto de la combustión no controlada. Este Material presente en el aire transporta microorganismos como bacterias, hongos y virus. (Quijano Parra, Quijano Vargas, \& Henao Martínez, 2010), por lo que han sido catalogadas como un importante foco en la generación de enfermedades respiratorias (Zosky et al, 2014).

Los hongos más comunes encontrados en el material particulado pertenecen a los géneros Cladosporium, Aspergillus, Penicillium, Alternaría, Mucor, Rhodotorula y algunos levaduriformes como Cryptococcus, Coccidioides, Blastomyces, Histoplasma (Underwood, 1992, Takahashi 1997; Mosso, Ullán, \& de la Rosa, 2002; Blanco, 2003). Estos producen enfermedades respiratorias como neumonías, micosis sistémicas, hipersensibilidad y micotoxicosis (Mosso et al, 2002), las cuales son más constantes en niños menores de 14 años y en adultos mayores, debido a que son individuos con respuesta inmune deficiente (Blanco, 2003; Gavidia, Pronczuk, \& Sly, 2009). 
Tabla 1: Especies y géneros de hongos transmitidos por el aire, que producen enfermedades en humanos.

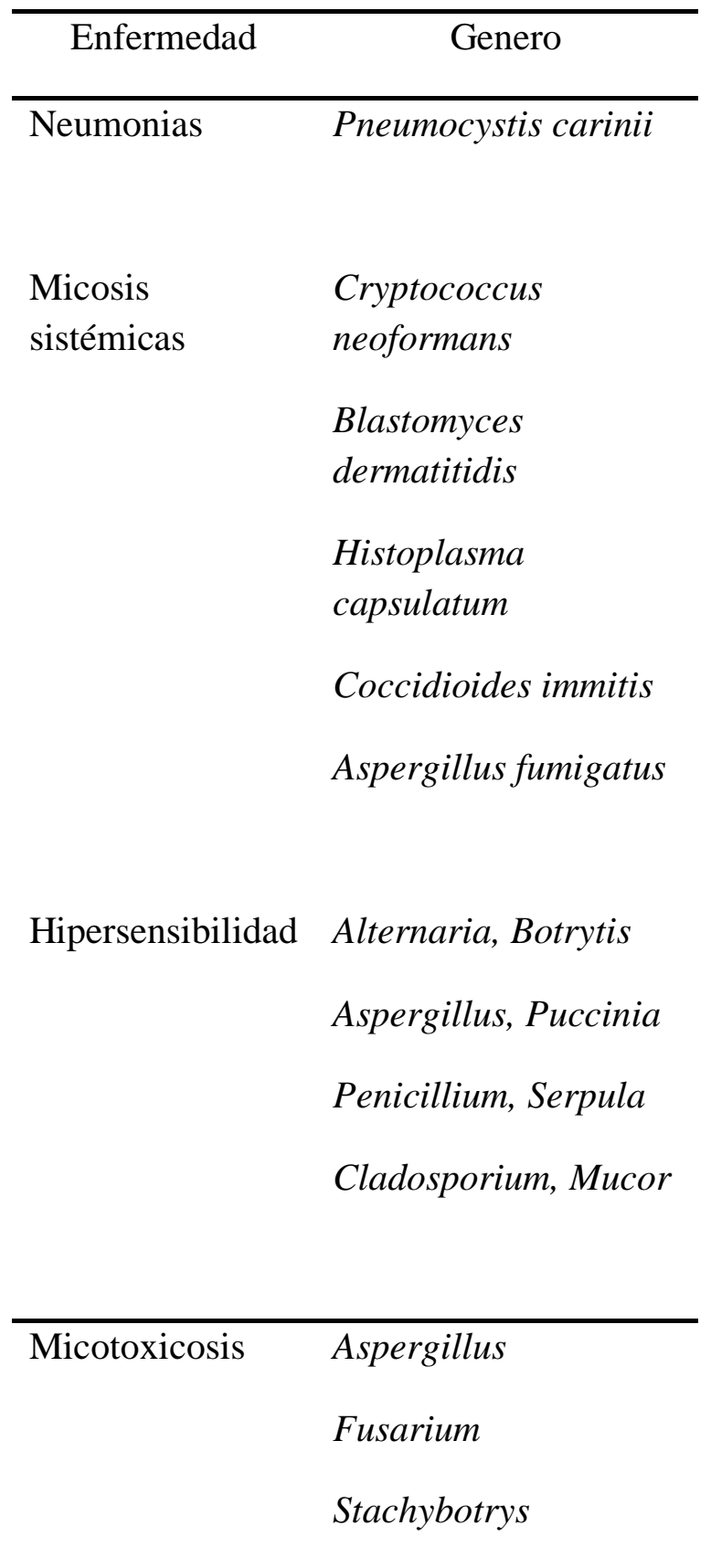

Fuente: El aire: hábitat y medio de transmisión de microorganismos. Mosso, Ullán, \& de la Rosa, 2002.
Solarte (1999) condujo un riguroso estudio en ciertas localidades de Bogotá, Bosa, Venecia, Trinidad Galán, Olaya Herrera y Engativá, las cuales se determinaron por ser zonas de influencia de las estaciones de monitoreo de calidad del aire del DAMA. De acuerdo a los resultados encontrados, se pudo concluir que las concentraciones de PM10 en los sitios analizados superan el valor máximo anual y diario permitido en la legislación internacional $\left(50 \mu \mathrm{g} / \mathrm{m}^{3}\right.$ y 150 $\mu \mathrm{g} / \mathrm{m}^{3}$ respectivamente), lo cual demuestra que los efectos adversos sobre la salud de los niños residentes en el sector pueden ser atribuidos al incumplimientos de dicho parámetro. También, se pudo analizar que un aumento de $10 \mu \mathrm{g} / \mathrm{m}^{3}$ en la concentración de PM10 produce un aumento de por lo menos el $8 \%$ en el número de consultas por enfermedad respiratoria en los niños menores de 14 años.

Blanco (2003) realizó una caracterización microbiológica del aire en la localidad de puerto Aranda, donde identificó microorganismos como Serratia sp, Klebsiella sp, Yersinia sp, Pseudomonas sp, Escherichia coli, Shigella sp, Corynebacterium $s p, \quad$ Candida sp, Rhodoturula sp, Aspergillus flavus, Aspergillus Níger, Penicillum sp, Staphylococcus aureus y Staphylococcus epidermis, sin identificar Haemophyllus influenzae y Streptococcus pneumonia que son los causantes más importantes de infecciones Respiratorias Agudas. 
Neva, Garcia, Libertad \& Yañez (2005) desarrollaron un estudio en México, con el fin de "determinar la concentración y tipo de microorganismos cultivables suspendidos en la atmósfera de la ciudad de Monterrey, por medio de análisis microbiológico de nueve sitios de la ciudad" en donde se tuvo en cuenta la intensidad de la actividad humana, el tráfico vehicular, la dirección del viento, la humedad relativa, y la temperatura a dos horas diferentes del día (9 am y 14 pm); durante esta investigación se realizaron conteos microbianos de bacterias mesofílicas aerobias, coliformes, hongos, cocos Gram Positivos como Staphylococcus aureus y un bacilo Gram Negativo la Pseudomona aeruginosa. Los cuales han sido clasificados como patógenos oportunistas y de alto riesgo para la salud de los seres humanos, ya que son causantes de alergias en ciertas épocas del año.

Otras investigaciones se realizaron en: Filadelphia (1973- 1988), Dallas (19901994), Búfalo (1988- 1990), Toronto (19701994), Ciudad de México (1990-1995), Londres (1987-1994), Birmingham (19921994), Róterdam (1983-1991), Roma (19921995) y Sao Paulo (1991-1992), con el objeto de conocer la relación entre concentraciones de material Particulado y los efectos que esta trae consigo a la salud de las personas, en dichos estudios se encontró una equivalencia proporcional entre estas dos variables, reportándose incrementos desde $0,6 \%$ en la tasa de mortalidad de $5 \%$ por cada $10 \mu \mathrm{g} / \mathrm{m}^{3}$ de aumento en la concentración de partículas
(Hoek, Brunekreef, Goldbohm, Fischer, \& Brandt, 2002).

\section{METODOLOGÍA}

El área de estudio se localizó en el perímetro del Casco Urbano del Municipio de Ocaña, ubicada al nororiente colombiano. Con coordenadas geográficas de $8^{\circ} 14^{\prime} 46^{\prime \prime} \mathrm{N}$ - 73⒉ $1^{\prime} 19^{\prime \prime} \mathrm{O}$, a una altura promedio de $1202 \mathrm{msnm}$. Para la toma de las muestras se utilizó la Red de Monitoreo de Calidad del Aire UFPSO - CORPONOR (Fig. 1), ubicada en tres puntos del municipio: Escuela de Bellas Artes de la Universidad Francisco de Paula Santander Ocaña, Avenida Francisco Fernández de Contreras y el barrio Santa Clara, conformada por tres muestreadores de partículas PM10 de alto volumen y dos estaciones meteorológicas. Se muestrearon tres filtros extraídos de cada una de las estaciones de la red de monitoreo, a los cuales se le tomaron cinco muestras al azar con dimensiones de $1 \mathrm{~cm}^{2}$ para un total de 15 muestras que se inocularon en medio de cultivo agar papa dextrosa (PDA), a $24^{\circ} \mathrm{C}$ por 15 días. Luego se aislaron las colonias para su respectiva identificación por medio de microscopia convencional, a través de la técnica de montaje en portaobjetos, donde se utilizó azul de lactofenol para la observación de las estructuras morfológicas de las colonias. 


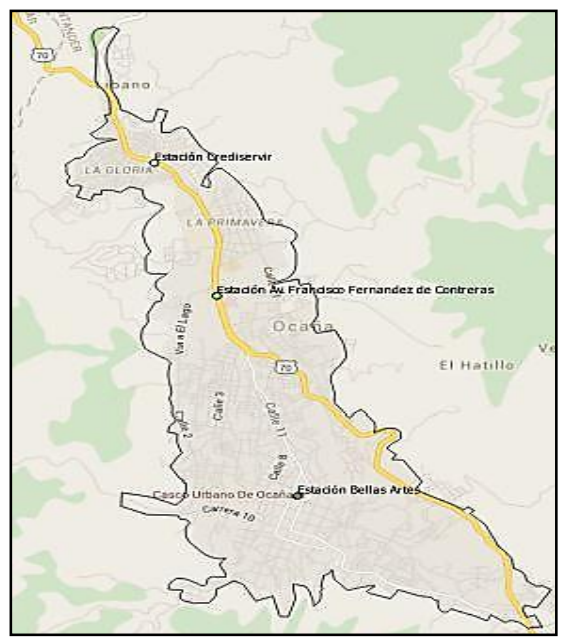

Fig. 1. Ubicación de las estaciones de la Red de Monitoreo de Calidad de Aire Universidad Francisco de Paula Santander Ocaña - CORPONOR Fuente: Autores.

\section{RESULTADOS}

Durante los días muestreados se observó que el índice de calidad de aire fue moderado en la estación de Santa Clara y bueno para las estaciones de la avenida Francisco Fernández de Contreras y Bellas Artes (Fig. 2).

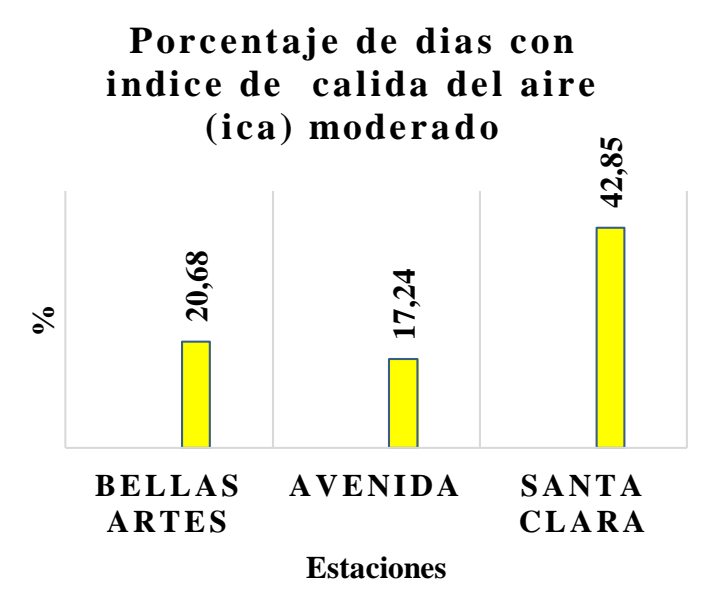

Fig. 2. Porcentaje del ICA para las tres estaciones. Fuente: Autores

Respecto a la carga micotica presente en el Material Particulado, se determinaron 3 géneros de hongos microscópicos y un grupo de hongos unicelulares (Tabla 2).

Tabla 2. Distribución de género de hongos identificados en cada estación de muestreo.

\begin{tabular}{llll}
\hline ESPECIES & $\mathbf{E}_{\mathbf{1}}$ & $\mathbf{E}_{\mathbf{2}}$ & $\mathbf{E}_{\mathbf{3}}$ \\
\hline $\begin{array}{l}\text { Penicillium } \\
\text { sp }\end{array}$ & 0 & 1 & 0 \\
$\begin{array}{l}\text { Aspergillus } \\
\text { niger }\end{array}$ & 1 & 1 & 1 \\
$\begin{array}{l}\text { Rhizopus sp } \\
\text { Levaduras }\end{array}$ & 1 & 0 & 0 \\
$\mathrm{E}_{1}=$ Estación Santa Clara \\
$\mathrm{E}_{2}=$ Estación Bellas Artes \\
$\begin{array}{l}\mathrm{E}_{3}=\text { Estación Av. Francisco Fernández } \\
\text { de Contreras. }\end{array}$
\end{tabular}

Fuente: Autores

Se observó que Aspergillus niger es la especie dominante ya que está presente en el material particulado de las tres estaciones de muestreo, la estación uno presentó mayor cantidad de especies asociados Rhizopus sp, Aspergillus niger y Levaduras. 
Por otro lado se obtuvo la información del Hospital Emiro Quintero Cañizares sobre las atenciones en el servicio de Urgencias Pediátricas, identificando las principales enfermedades respiratorias que afectan a la salud de la población infantil Ocañera (Tabla 3) en el periodo comprendido entre diciembre de 2014 hasta marzo de 2015, de acuerdo a lo anterior, la bronquitis es la enfermedad respiratoria, que más afecta a la salud de los niños ocañeros seguida de la bronconeumonía.

Tabla 3. Enfermedades respiratorias consultadas en Urgencias Pediátricas de la ESE HEQC.

\begin{tabular}{lll}
\hline Enfermedad & $\begin{array}{l}\mathbf{N}^{\circ} \\
\text { Consultas }\end{array}$ & $\begin{array}{l}\text { Porcentaje } \\
(\%)\end{array}$ \\
\hline Asma & 5 & 12,15 \\
Bronquitis & 58 & 54,20 \\
Bronconeumonía & 20 & 18,69 \\
Neumonía & 7 & 6,54 \\
I.R.A. & 4 & 3,73 \\
Infecciones de & 5 & 4,67 \\
vías respiratorias & & \\
\hline
\end{tabular}

Fuente: Autores

\section{DISCUSIÓN}

Según la EPA, en las zonas que presentan calidad de aire moderado, se observa que los niños y adultos son sensibles, especialmente los que sufren de enfermedades respiratorios como el asma (Bruni Dávila, 2004). La estación Santa Clara, es la zona de mayor preocupación en la calidad del aire, con una clasificación de moderado, y que además presenta el mayor número de géneros de hongos según las muestras tomadas. Sin embargo no se puede establecer una correlación entre el material particulado y la producción de enfermedades respiratorias debido a que no se cuenta con los registros históricos que relacione los dos parámetros.

Murray, Rosenthal, \& Pfaller (2009) en su libro Microbiología Médica, afirman que el género Aspergillus está involucrado en infecciones de vías respiratorias superiores como la Sinusitis y también, provoca infecciones pleuropulmonares y bronquiales, comprendidas en este grupo, la bronquitis, la neumonía, aspergiloma (masa de hongos) y la empiema. Además establecieron una relación del Aspergillus niger con la otitis externa. No obstante, el Instituto Nacional de Seguridad e higiene en el Trabajo (2012) señala que este género de hongo, produce en el ser humano efectos alérgicos como el asma, rinitis y alveolitis alérgica extrínseca o neumonitis por hipersensibilidad que puede desarrollarse después de la exposición a sus conidios.

Raper \& Fennell (1973) Establecieron que A. fumigatus, A. flavus, A. niger y A. terreus, son las 4 especies de Aspergillus causantes de la mayoría de las enfermedades respiratorias. El pulmón y los senos paranasales se constituyen como los principales conductos para el Aspergillus (Diaz Sanchez \& Lopez Viña, 2004), y sus esporas pueden estar suspendidas en el aire durante un tiempo considerable, contaminando cualquier área que esté presente con este tipo de medio gaseoso alterado.

En cuanto, al Penicillium se ha comprobado que este género tiene la capacidad de ocasionar alveolitis. (Lacey, 1973), (Chen, 1970).

\section{CONCLUSIONES.}

La concentración de Material Particulado (PM10), en Ocaña, Norte de Santander de acuerdo a los datos suministrados por la Red 
de Monitoreo y Vigilancia de la Calidad del Aire UFPSO-CORPONOR ha venido afrontando un incremento en estos últimos meses, a pesar de que el municipio no se caracteriza por la actividad industrial, pero si, por el sector comercial, el cual hace que por el territorio halla un gran flujo de fuentes móviles que provocan este aumento de partículas suspendidas en el aire.

La zona de mayor preocupación en Ocaña, en cuanto a la calidad del Aire, es el área de influencia que tiene la estación Santa Clara, ya que presenta una calidad del aire moderado en relación con las otras dos zonas de estudio (Avenida y Bellas Artes) teniendo en cuenta que en los filtros de esta estación, fue en donde se encontró el mayor número de géneros de hongos presentes en el Material Particulado (PM10), que tienen teóricamente estrecha relación con enfermedades respiratorias por considerarse algunos de ellos como patógenos oportunistas. (Blanco, 2003). Sin embargo no se puede hacer una correlación entre estos dos parámetros.

Por otro lado, la bronquitis es la enfermedad más padecida por la población infantil en el municipio de Ocaña, siendo la enfermedad que más atiende el servicio de urgencias pediátricas de la ESE Hospital Emiro Quintero Cañizares, y que de acuerdo a la literatura es relacionada con el Aspergillus sp.

\section{FINANCIACIÓN}

La Universidad Francisco de Paula Santander Ocaña, a través del laboratorio de calidad de aire y el laboratorio de biotecnología vegetal, apoyó la realización de la presente investigación.

\section{BIBLIOGRAFÍA}

Arteaga, J., \& Duran, H. (2001). Contaminación atmosférica en Chile: Antecedentes y políticas para su control. Medio ambiente, ecología $y$ salud pública. Obtenido de Universidad de Santiago. Instituto Medio Ambiente. : http://www. usach

Barrios, C., Peña Cortés, F., \& Osses Bustingorry, S. (2004). Efectos de la Contaminación Atmosférica por Material Particulado en las enfermedades respiratorias aguas en menores de 5 años. Ciencia $y$ Enfermeria, 25.

Blanco, L. (2003). Caracterización microbiolgica del Material Particulado como factor de riesgo sobre la salud en la localidad de Puente Aranda, Bogota. Universidad de la Salle.

Brauer, M., Bartlett, K., Regalado Pineda, J., \& Perez Padilla, R. (1996). Assessment of Particulate Concentrations from Domestic Biomass Combustion in Rural Mexico. . Environ. Sci. Technol. , 30, 1, 104- 109.

Bruni Dávila, H. A. (2004). Resultados del Monitoreo de material particulado. San Francisco, USA: U.S. Environmental Protection Agency. Obtenido de http://yosemite.epa.gov/R9/SFUND/ R9SFDOCW.NSF/3dc283e6c5d605 6f88257426007417a2/27345cb8d71e f845882577ce005be962/\$FILE/McF arland\%207_04_Spanish.pdf 
Chen, C. Y. (1970). Seasonal Variation of fungi in the sputa and throats os asthmatic patients. J. Form. Med.Ass. .

Diaz Sanchez, C., \& Lopez Viña, A. (2004). Aspergillus y Pulmón . Ach Bronconeumol, Vol 40 Num 03.

García, F., Agudelo, R., \& Jiménez, K. (2006). Distribucción espacial y temporal de la concentración de material particulado en Santa Marta, Colombia. Revista Facultad Nacional de Salud Pública, 24 (2), 73-82.

Gavidia, T., Pronczuk, J., \& Sly, P. (2009). Impactos ambientales sobre la salud respiratoria de los niños: Carga global de las enfermedades respiratorias pediatricas ligadas al ambiente. . Revista Chilena de enfermedades respiratorias, 25 ("), 99-108.

HEQC, E. (2015). Empresa Social del Estado Hospital Emiro Quintero Cañizares. Ocaña, Norte de Santander.

Hoek, G., Brunekreef, B., Goldbohm, S., Fischer, P., \& Brandt, P. (2002). Asociation between mortality and indicators of traffic-related air pollution in the Netherlands. The Lancet.

Instituto Nacional de Seguridad e higiene en el Trabajo. (23 de Septiembre de 2012). DATABiO. Fichas de Agentes Biologicos: Aspergillus spp.
Obtenido de http://www.insht.es/RiesgosBiologic os/Contenidos/Fichas\%20de\%20age ntes\%20biologicos/Fichas/Hongos/Fi cha\%20Aspergillus\%20spp.pdf

Lacey, J. (1973). The air spora of a portuguesa cork factory . Ann. occup.

Lopez, C. E. (s.f.). Enfermedades causadas por hongos.

Mosso, M., Ullán, C., \& de la Rosa, M. (2002). El aire: habitat y medio ambiente de transmision de microorganismos. Observatorio Medioambiental, Vol. 5: 375-402.

Murray, P., Rosenthal, K., \& Pfaller, M. (2009). Microbología Médica. Barcelona, España: Elsevier España .

Oyala, D., \& Perez, F. (2006). Caracterización cualitativacuantitativa de bioaerosoles relacionados con factores meteorologicos y material particulado en Puente Aranda Bogota D.C. Bogota: Universidad de la Salle.

Quijano Parra, A., Quijano Vargas, M., \& Henao Martínez, J. (2010). Caracterización fisicoquímica del material Particulado fracción respirable PM2. Bistua: Revista de la Facultad de Ciencias Básicas. Universidad de Pamplona.

Raper, KB; Fennell, DI;. (1973). The genus Aspergillus. Huntington: Roberth E, Krieger Pub. 
Samara, C., \& Voutsa, D. (2005). Size distribution of airborne particulate matter andassociated heavy metals in the roadside environment. Journal Chemosphere, 59, 1197-1206.

Solarte, I. (1999). Contaminación atmósferica y enfermedades respiratoria en menores de 14 años en Bogota. . Bogota: Universidad Javeriana.

Zosky, G., Boylen, C., Wong, R., Smirk, M., Gutiérrez, L., Woodward, R., . . . Cook, A. (2014). Variability and consistency in lung inflammatory responses to particles with a geogenic origin. Respirology: official journal of the Asian Pacific Society of Respirology. 